\title{
$\beta$-catenin deacetylation is essential for WNT-induced proliferation of breast cancer cells
}

\author{
SHAO-HUA WANG, NING LI, YAO WEI, QIU-RONG LI and ZE-PING YU
}

Department of General Surgery, Jinling Hospital, Nanjing University School of Medicine, Nanjing, Jiangsu 210002, P.R. China

Received May 22, 2013; Accepted December 10, 2013

DOI: $10.3892 / \mathrm{mmr} .2014 .1889$

\begin{abstract}
Deregulation of the WNT signaling pathway is associated with the development and progression of breast cancer. $\beta$-catenin mutations have been found to constitutively activate $\beta$-catenin-T-cell factor (TCF) signaling in other types of cancer. $\beta$-catenin acetylation regulates $\beta$-catenin-TCF4 interaction in WNT signaling, but it remains unknown whether the acetylation of $\beta$-catenin is involved in WNT-induced proliferation of breast cancer cells. In this study, a lower level of acetylated $\beta$-catenin (K345) was observed in breast cancer tissues. WNT3A stimulated the downregulation of $\beta$-catenin acetylation and promoted the proliferation of MCF7 cells. The K345Q mutation in $\beta$-catenin inhibited WNT-induced cell growth and axin2/TCF7 upregulation in breast cancer cells. By contrast, K345R mutants could mimic deacetylated $\beta$-catenin to generate the WNT-elicited phenotype. Additionally, the acetylation of $\beta$-catenin may prime $\beta$-catenin for phosphorylation. Further investigation revealed that the deacetylase HDAC6 was responsible for WNT-induced deacetylation of $\beta$-catenin in breast cancer cells. In conclusion, the epigenetic modification of $\beta$-catenin may be essential for WNT signaling in breast cancer progression, and blocking the occurrence of $\beta$-catenin acetylation may provide a novel therapeutic approach for breast cancer.
\end{abstract}

\section{Introduction}

Breast cancer is the most frequently diagnosed type of cancer and the leading cause of cancer-related mortality in females. It accounts for $\sim 23 \%$ of total cancer cases worldwide (1). Metastatic breast cancer often occurs several years after primary breast cancer resection and it is responsible for the majority of breast cancer-related fatalities (2). Until recently, the prognosis of breast cancer and the understanding of the pathogenesis of this disease have been relatively poor.

Correspondence to: Dr Ning Li, Department of General Surgery, Jinling Hospital, Nanjing University School of Medicine, 305 East Zhongshan Road, Nanjing, Jiangsu 210002, P.R. China

E-mail: lining1106pubsci@163.com

Key words: WNT, acetylation, $\beta$-catenin, P300, breast cancer
Aberrant activation of the WNT signaling pathway is vital in the development of numerous types of human cancer and the canonical WNT/ $\beta$-catenin pathway participates in several biological processes, such as cell proliferation and differentiation (3). The secreted WNT ligands stimulate intracellular signaling transduction, which leads to the stabilization and nuclear translocation of the key effector $\beta$-catenin. $\beta$-catenin then binds to lymphoid enhancer-binding factor-1/T-cell factor (TCF) and activates target gene transcription $(3,4)$. Deregulation of the WNT pathway frequently occurs in multiple types of cancer and underlies hereditary syndromes (5). Mutations in adenomatous polyposis coli (APC), axin or $\beta$-catenin activate the deregulated WNT signaling pathway and promote the transcription of target genes encoding colorectal cancer-associated proteins (6). However, WNT pathway mutations are rarely detected in breast cancer (7), and deregulation of WNT signaling occurs by autocrine mechanisms $(8,9)$. Autocrine WNT signaling has been proposed to promote breast cancer cell motility and proliferation via the canonical WNT pathway and epidermal growth factor receptor transactivation $(9,10)$.

$\beta$-catenin is a central regulator of the WNT signaling pathway (11) and it can also act as a co-activator through interaction with other factors to regulate downstream gene expression (12). Furthermore, the epigenetic modification of $\beta$-catenin is an important mode of regulation of $\beta$-catenin stability and cellular location, as well as its transcriptional activity $(13,14)$. Without WNT stimulation, glycogen synthase kinase 3 phosphorylates $\beta$-catenin and triggers its ubiquitination-depedent degradation (15-17). $\beta$-catenin has also been found to be acetylated by acetyltransferase CREB-binding protein/p300 at different residues (K49 and K345) $(18,19)$, and the deacetylation of $\beta$-catenin by HDAC6 is involved in the interferon regulatory factor 3 (IRF3) signaling pathway (20). However, the association between epigenetic modification of $\beta$-catenin and autocrine WNT signaling is unclear in breast cancer.

In the present study, the protein level of acetylated $\beta$-catenin was found to be lower in breast cancer tissues compared with the adjacent normal tissues. In the MCF7 breast cancer cell line, WNT downregulated the acetylation level of $\beta$-catenin and enhanced the proliferation of breast cancer cells, which was inhibited by the K345Q mutation in $\beta$-catenin. Conversely, the K345R deacetylation mimic mutant exhibited an increased proliferation rate of breast 
cancer cells. WNT-induced axin 2 and TCF7 upregulation was also influenced by the deacetylation of $\beta$-catenin. Further investigation revealed that HDAC6 may be responsible for this process. These results provide novel insights for breast cancer therapy through targeting $\beta$-catenin deacetylation to regulate WNT pathway activity.

\section{Materials and methods}

Samples and cell culture. Five samples of breast cancer tissue and the adjacent normal tissue were obtained at the time of surgery from five patients with breast cancer who were treated at the Department of Surgery, Nanjing Jinling Hospital (Nanjing, China). MCF7 cells were maintained in RPMI-1640 medium (Invitrogen Life Technologies, Carlsbad, CA, USA) supplemented with $10 \%$ fetal calf serum.

Cell fractionation. WNT3A-stimulated MCF7 cells were washed and collected with cold phosphate-buffered saline (PBS). Cell pellets were then resuspended in hypotonic lysis buffer [10 mM KCl, $10 \mathrm{mM}$ Tris (pH 7.5) and 2 mM EDTA] containing protease inhibitor and phosphatase inhibitor cocktail tablets (Roche Applied Science, Indianapolis, IN, USA) and histone deacetylase inhibitor cocktail (including trichostatin A, negative allosteric modulator, suberoylanilide hydroxamic acid and MS275). Cell suspensions were incubated on ice for $30 \mathrm{~min}$. Nuclear proteins, including the unlysed cells, were pelleted by centrifugation at $400 \mathrm{x} \mathrm{g}$ for 2 min at $4^{\circ} \mathrm{C}$. The supernatant that contained cytoplasm and membrane proteins was then centrifuged at 17,500 x $\mathrm{g}$ for $30 \mathrm{~min}$ at $4^{\circ} \mathrm{C}$. The supernatant was collected and analyzed by western blotting. Anti-Phospho- $\beta$-catenin S33/S37/T41 (1:1,000; Cell Signaling Technology, Inc., Beverly, MA, USA), anti-Ace- $\beta$-catenin K345 antibody (1:500; Santa Cruz Biotechnology,Inc.,SantaCruz,CA,USA), anti-Ace- $\beta$-catenin K49 antibody (1:400; Cell Signaling Technology, Inc.), anti$\beta$-catenin (1:1,000; BD Transduction Laboratories, San Jose, CA, USA) and anti-GAPDH (1:10,000; Abcam, Cambridge, MA, USA) were used for immunoprecipitation or western blot analysis.

RNA isolation and quantitative polymerase chain reaction $(q P C R)$. Total RNA was isolated from MCF7 cells with TRIzol (Invitrogen Life Technologies). Total RNA $(2.5 \mu \mathrm{g})$ was used for cDNA synthesis using Superscript II (Invitrogen Life Technologies), according to the manufacturer's instructions. Each PCR cycle was conducted in a volume of $25 \mu \mathrm{l}$ reaction mixture (HotStarTaq Master mix; Qiagen, Hilden, Germany). The reverse-transcribed cDNA products were directly analyzed by qPCR using SYBR-Green (Sigma, Oakville, ON, Canada) as a label. The primers were used as follows: Sense: 5'-GAAGGTGAAGGTCGGAGT-3' and antisense: 5'-GAAGATGGTGATGGGATTTC-3' for GAPDH; sense: 5'-TACACTCCTTATTGGGCGATCA-3' and antisense: 5'-TTGGCTACTCGTAAAGTTTTGGT-3' for axin2; and sense: 5'-TGGAGGGCTCTTTAAGGGG-3' and antisense: 5'-GATCCGTTGGGGAGGTAGG-3' for TCF7.

In vitro tumor cell growth assay. The in vitro growth of MCF7 cells was determined by measuring increases in cell number using the 3-(4,5-dimethylthiazol-2-yl)-2,5-diphenyltetrazolium bromide assay (Promega Corporation, Madison, WI, USA) according to the manufacturer's instructions. Three independent experiments were performed. Cell-mediated reaction products were then recorded by a enzyme-linked immunosorbent assay reader (SpectraMax 250; Molecular Devices, Sunnyvale, CA, USA) at a wavelength of $450 \mathrm{~nm}$.

Immunoprecipitation. At $80 \%$ confluence, MCF7 cells were treated with either WNT3A or control-conditioned medium as indicated. Cells were washed and collected with cold PBS, lysed in cold lysis buffer containing $150 \mathrm{mM} \mathrm{NaCl}, 30 \mathrm{mM}$ Tris (pH 7.5), 1 mM EDTA, $1 \%$ Triton X-100, 10\% glycerol, $0.1 \mathrm{mM}$ phenylmethylsulfonyl fluoride, $0.5 \mathrm{mM}$ dithiothreitol, protease inhibitor cocktail tablets (EDTA-free) and phosphatase inhibitor cocktail tablets (both from Roche Applied Science, Indianapolis, IN, USA) and histone deacetylase inhibitor cocktail (including TSA, NAM, SAHA and MS275). Following separation by centrifugation (14,000 rpm for $30 \mathrm{~min}$ at $4^{\circ} \mathrm{C}$ ), the cellular lysates were precleared with IgG-agarose beads (Sigma, St. Louis, MO, USA) for at least $6 \mathrm{~h}$ at $4^{\circ} \mathrm{C}$. Immunoprecipitation of endogenous $\beta$-catenin was conducted by incubating the cellular lysates with anti- $\beta$-catenin antibody, using mouse IgG (BD Transductions Laboratories, Lexington, KY, USA) as a blank control. Immunoproteins were washed with cold lysis buffer six times, resuspended in $2 X$ SDS sample buffer, and subjected to SDS-PAGE and western blot analysis.

HDAC6 knockdown and transfection. The synthesized HDAC6 and control (scramble) small hairpin RNAs (shRNAs) were cloned into pSuper vectors (Oligoengine, Seattle, WA, USA). The HDAC6 knockdown efficiency was determined by western blot analysis. HDAC6 shRNAs sequences were shown as follows: 5'-TACAACAGCC ACAACGTCTAT-3' for control shRNA; and 5'-CATCCCATC CTGAATATCCTT-3' for HDAC6 shRNA. Plasmids were transfected with Lipofectamine 2000 (Invitrogen Life Technologies) into MCF7 cells at $50 \%$ confluency.

Statistical analysis. Each experiment was performed at least three times. Data are presented as the mean \pm standard deviation. Statistical significance was determined by Student's t-test and $\mathrm{P}<0.05$ was considered to indicate a statistically significant difference.

\section{Results}

$\beta$-catenin acetylation is downregulated in breast cancer tissues. Aberrant WNT signaling is activated in breast cancer in an autocrine manner to regulate breast cancer cell proliferation (9). To investigate the association between the epigenetic modification of $\beta$-catenin and breast cancer, the protein level of acetylated $\beta$-catenin was analyzed by western blotting in five samples of breast cancer tissue and the corresponding adjacent normal tissue (Fig. 1). The results showed that the level of acetylated $\beta$-catenin at the K345 site in the breast cancer tissues was markedly lower than in normal tissues. Notably, K49 acetylated $\beta$-catenin was maintained at a relatively stable level. This suggests that the modification of $\beta$-catenin at the 


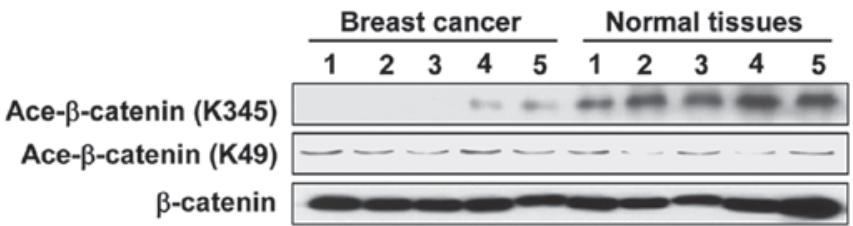

Figure 1. $\beta$-catenin acetylation is downregulated in breast cancer tissues. The protein levels of acetylated $\beta$-catenin at K345 and K49 were examined by western blot analysis with K345 and K49 acetylation-modified residue-specific antibodies in five samples of breast cancer tissue and the corresponding adjacent normal tissue.

A

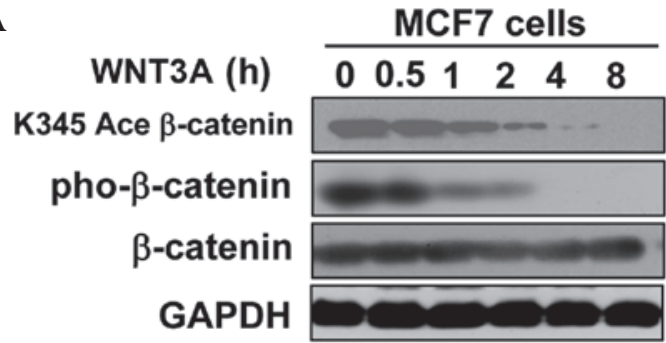

B

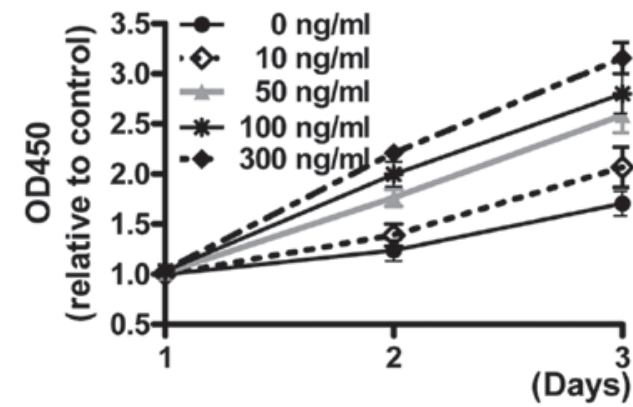

C

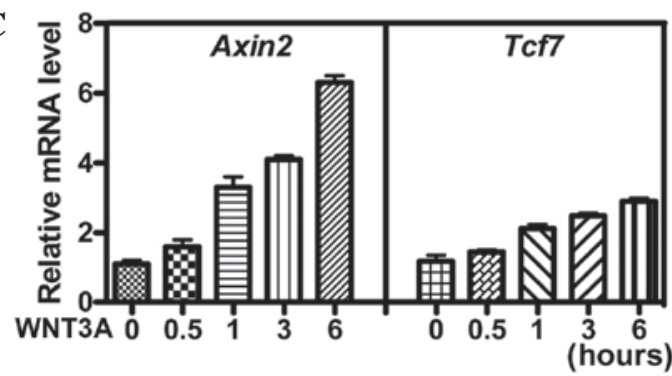

Figure 2. WNT3A inhibits $\beta$-catenin acetylation and promotes breast cancer cell proliferation. (A) MCF7 cells were treated with WNT3A $(50 \mathrm{ng} / \mathrm{ml})$ for 0.5-8 $\mathrm{h}$ and western blot analysis was performed to determine the protein levels of acetylated (Ace) $\beta$-catenin (K345), phosphorylated (pho) $\beta$-catenin, total $\beta$-catenin and GAPDH. (B) MCF7 cells were stimulated with $0,10,50$

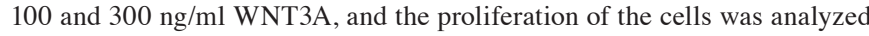
on different days by optical density (OD) 450 values normalized to control. (C) Quantitative polymerase chain reaction analysis of the expression of axin2 and T-cell factor 7 (TCF7) when MCF7 cells were treated with WNT3A $(50 \mathrm{ng} / \mathrm{ml})$ for $0.5-6 \mathrm{~h}$.

K345 site may be associated with the development of breast cancer.

WNT signaling decreases the $\beta$-catenin acetylation level and promotes breast cancer cell proliferation. The acetylation level of $\beta$-catenin is downregulated in breast cancer, while the association between $\beta$-catenin deacetylation and autocrine
WNT signaling remains unknown. To explore this question, the MCF7 breast cancer cell line was used as an in vitro model. In MCF7 cells, WNT3A treatment downregulated the acetylation level of $\beta$-catenin (K345) in a time-dependent manner (Fig. 2A). The proliferation rate of MCF7 cells was futher determined under the stimulation of WNT3A and it was found that WNT promoted the proliferation of the breast cancer cells in a dose-dependent manner (Fig. 2B), which is consistent with a previous study (9). The expression level of WNT target genes axin2 and TCF7 $(21,22)$ was examined by qPCR and the results showed that the mRNA level was markedly elevated by WNT treatment (Fig. 2C). These observations suggest that WNT-induced $\beta$-catenin deacetylation and cell proliferation may be associated.

K345 mutants affect the growth of breast cancer cells by influencing $\beta$-catenin phosphorylation. $\beta$-catenin acetylation has been proposed as a novel mechanism for the regulation of WNT/ $\beta$-catenin transcriptional activity (19); therefore the effects of K345 mutation on the epigenetic modification of $\beta$-catenin in $293 \mathrm{~T}$ cells, in which the transfected $\beta$-catenin can be normally acetylated, were examined (Fig. 3A). Two $\beta$-catenin mutants (K345R and K345Q), utilized to mimic acetylation modification, were transfected into the MCF7 cells. K345R mutants inhibited the acetylation of $\beta$-catenin and impaired WNT-induced downregulation of $\beta$-catenin phosphorylation in 293 T cells (Fig. 3A). Conversely, the K345Q mutation, which could mimic the acetylation modification, promoted the phosphorylation of $\beta$-catenin even in the presence of WNT3A (Fig. 3B). These results demonstrated that the acetylation at residue K345 may be the triggering signal for $\beta$-catenin phosphorylation, which can be used to mark the molecule for subsequent degradation.

It was assessed whether the K345 mutation in $\beta$-catenin affects WNT-induced cell proliferation and WNT responsive gene expression in MCF7 cells. The deacetylation mutation K345R promoted the proliferation of the breast cancer cells (Fig. 3C), similar to the effects of WNT3A (Fig. 2B). The acetylation mimic mutant K345Q suppressed the growth of MCF7 cells and also impaired the promotional effects of WNT3A on the proliferation rate of breast cancer cells (Fig. 3C). In addition, the expression of WNT-responsive genes axin2 and MCF7 was upregulated in K345R mutants and decreased in K345Q mutants (Fig. 3D). It was hypothesized that $\beta$-catenin undergoes acetylation and phosphorylation as well as further degradation without WNT stimulation, and that WNT pathway activation could promote the deacetylation of $\beta$-catenin and the downregulation of phosphorylation, and subsequently activate the canonical $\mathrm{WNT} / \beta$-catenin pathway.

HDAC6 is responsible for the WNT-induced decrease in $\beta$-catenin acetylation in breast cancer cells. Previously, HDAC6 has been proposed as a requirement for $\beta$-catenin deacetylation on Lys-49 and its nuclear translocation in the epidermal growth factor receptor or the interferon regulatory transcription factor 3 signaling pathway $(20,23)$. However, it is unknown whether the deacetylation of $\beta$-catenin on Lys-345 is also exerted by HDAC6. Thus an immunoprecipitation assay was performed using an anti- $\beta$-catenin antibody and a strong 
$\mathbf{A}$

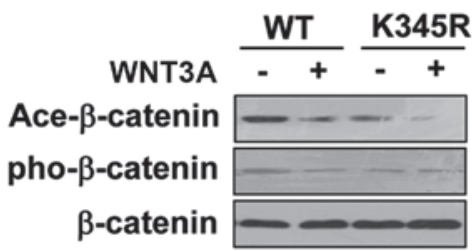

C

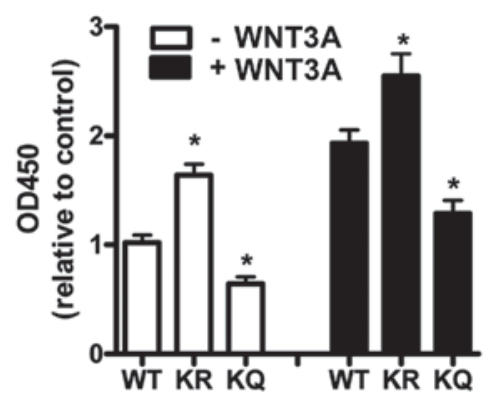

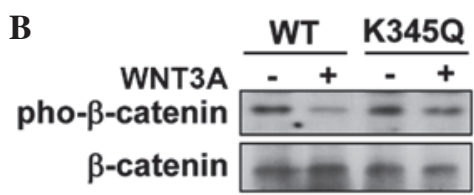

D

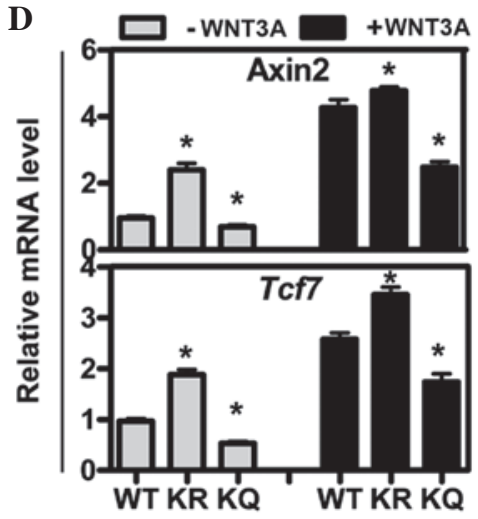

Figure 3. K345 mutants affect the growth of breast cancer cells through $\beta$-catenin phosphorylation. (A) Wild-type $\beta$-catenin (WT) and K345R (KR) mutants were transfected into $293 \mathrm{~T}$ cells with or without WNT3A $(50 \mathrm{ng} / \mathrm{ml}$ ) treatment. The levels of K345-acetylated (Ace) $\beta$-catenin, phosphorylated (pho) $\beta$-catenin and total $\beta$-catenin were determined by western blot analysis. (B) WT and K345Q (KQ) mutants were transfected into 293T cells with and without WNT3A $(50 \mathrm{ng} / \mathrm{ml})$ stimulation. Whole cell lysates were analyzed for the protein levels of phosphorylated (pho) $\beta$-catenin and total $\beta$-catenin. (C) MCF7 cells were transfected with WT, KR and KQ mutants, and the proliferation of these cells was analyzed 48 h post-transfection by optical density (OD) 450 values with and without WNT3A $(50 \mathrm{ng} / \mathrm{ml})$ treatment. (D) Quantitative polymerase chain reaction analysis of the expression of axin2 and T-cell factor 7 (TCF7) in WT, KR and KQ-transfected MCF7 cells with and without WNT3A (50 ng/ml) stimulation.
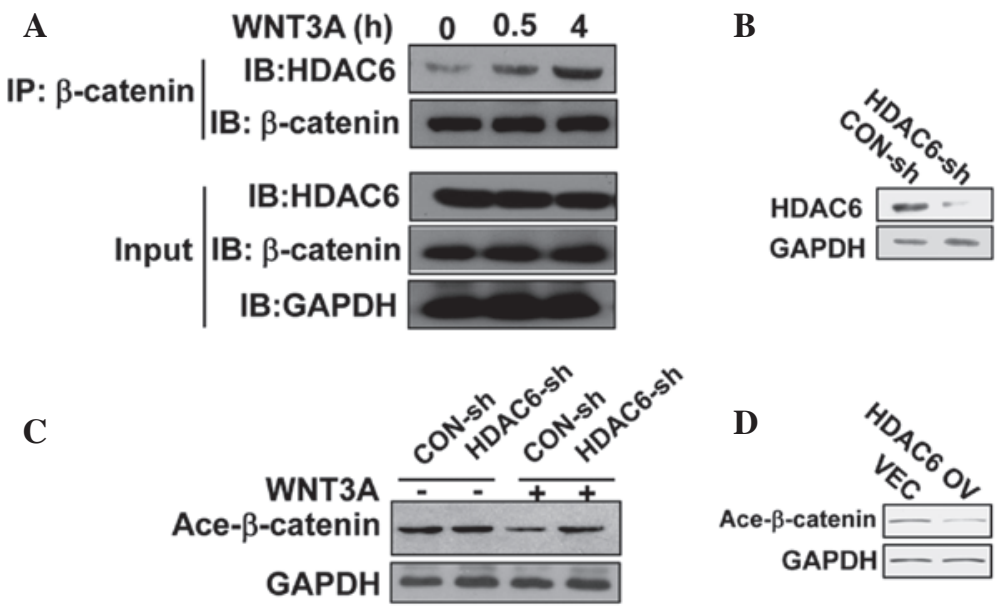

Figure 4. HDAC6 is responsible for WNT decreased $\beta$-catenin acetylation in breast cancer cells. (A) $\beta$-catenin immunoprecipitates with HDAC6. Cell lysates from MCF7 cells $\left(2 \times 10^{6}\right.$ cells) with or with WNT3A stimulation were immunoprecipitated with an anti- $\beta$-catenin antibody. The immunoprecipitate and input control samples were analyzed by western blotting with anti-HDAC6, anti- $\beta$-catenin and anti-GAPDH antibodies. (B) Control shRNA (CON-sh) or HDAC6 shRNA (HDAC6-sh) expressing plasmids were transfected into MCF7 cells and knockdown efficiency were determined by western blot analysis with anti-HDAC6 antibody. (C) Protein analysis of acetylated $\beta$-catenin in CON-sh or HDAC6-sh transfected MCF7 cells with or without WNT3A (50 ng/ml) treatment. (D) The protein level of acetylated $\beta$-catenin was determined when MCF7 cells were transfected with pCDNA3 vector (VEC) or HDAC6 overexpression plasmids.

interaction between endogeous HDAC6 and $\beta$-catenin under the stimulation of WNT3A was observed, indicating that the two proteins were present in a complex (Fig. 4A). In order to establish the link between HDAC6 and $\beta$-catenin, HDAC6 shRNA was delivered into MCF7 cells, which efficiently knocked down the expression of HDAC6 at the protein level (Fig. 4B). Without WNT treatment, HDAC6 knockdown marginally enhanced the acetylation level of $\beta$-catenin (K345).
However, WNT-induced downregulation of the acetylated $\beta$-catenin level was significantly rescued by HDAC6 shRNA (Fig. 4C), suggesting that HDAC6 affects the acetylation level of $\beta$-catenin. To further confirm this, HDAC6 was overexpressed in MCF7 cells, which resulted in the reduction of the acetylated $\beta$-catenin level (Fig. 4D). In conclusion, HDAC6 is sufficient and required for the deacetylation of $\beta$-catenin in breast cancer cells. 


\section{Discussion}

Aberrant activation of WNT signaling frequently occurs in numerous types of human cancer and targeting this pathway may be a promising therapeutic approach (24). The deregulation of the WNT signaling pathway can occur by different mechanisms, including APC/ $\beta$-catenin mutations in colorectal cancer and autocrine WNT signaling in breast cancer $(8,9,25)$. Protein post-translational modifications have also been reported in human cancers. Previously, the global loss of monoacetylation and trimethylation of histone $\mathrm{H} 4$ has been established as a common hallmark of human cancer cells (26), demonstrating that cancer progression is associated with deregulation of biological events at the transcriptional level as well as the post-translational level. In the present study, it was demonstrated that the protein level of acetylated $\beta$-catenin (K345) is markedly lower in breast cancer tissues than in the adjacent normal tissues (Fig. 1). It is possible that $\beta$-catenin is deacetylated during the progression of breast cancer. Although the quantity of specimens used in the present study was limited, the data indicate that the epigenetic modification of $\beta$-catenin may be correlated with breast cancer.

Autocrine WNT activity in human breast cancer cells with diverse genetic alterations has been identified in a previous study (9). In the present study, the possibility that the downregulation of acetylated $\beta$-catenin level is caused by WNT ligands was analyzed in MCF7 cells. As expected, WNT3A stimulation leads to a reduction in the acetylated and phosphorylated $\beta$-catenin levels. Consistent with previous findings (9), WNT signaling promoted the proliferation capacity of breast cancer cells in a dose-dependent manner. In addition, the expression of two important WNT downstream targets, axin2 and TCF7, was markedly elevated under the WNT3A treatment (Fig. 2), which may result in autocrine-activated WNT signaling in the nucleus to promote cell proliferation in breast cancer.

In the present study, the alterations of residue-specific acetylation of $\beta$-catenin in breast cancer cells was notable. The level of acetylated $\beta$-catenin at K345 was decreased in cancer tissues whereas K49-acetylated $\beta$-catenin was expressed at a relatively stable level. Previous studies show that the mutation of $\beta$-catenin at K49 neither impaired the WNT-responsive reporter TOPFLASH transactivation nor modulated the interaction of $\beta$-catenin with TCF $(18,19)$. Conversely, the K345A mutation abolished the interaction between $\beta$-catenin and TCF4, and K345 mutations affected their ability to transactivate the TOPFLASH reporter (19).

To explore the functional association between $\beta$-catenin acetylation and WNT signaling in breast cancer, the effects of K345 mutated $\beta$-catenin were examined with and without WNT treatment. The K345R mutation repressed the acetylation and phosphorylation of $\beta$-catenin, and also promoted the proliferation of MCF7 cells. Furthermore, K345Q $\beta$-catenin mutants antagonized WNT-induced downregulation of phosphorylated $\beta$-catenin and increased the cell proliferation rate (Fig. 3). The change in the phosphorylation level in K345-mutated $\beta$-catenin suggests that the phosphorylation of $\beta$-catenin may be dependent on its acetylation step. This view is supported by previous data establishing the link between phosphorylation and acetylation in PRKAA1 (27), and explains how K345A (similar to K345Q) $\beta$-catenin was shown to be efficiently degraded by APC (28). K345Q $\beta$-catenin mutants may constitutively phosphorylate $\beta$-catenin, lead to its degradation and further inhibit WNT signaling activity. The marginal downregulation of total $\beta$-catenin is observed in Fig. 3B. In previous studies $(20,23)$, HDAC6 has been identified as the deacetylase that regulates the aectylation level of $\beta$-catenin. HDAC6 is sufficient and required for WNT-stimulated deacetylation of $\beta$-catenin in breast cancer cells, but the specific correlation between HDAC6 and WNT signaling requires further investigation.

In conclusion, the present study demonstrated that downregulation of the level of acetylated $\beta$-catenin is associated with breast cancer. In vitro assays in MCF7 breast cancer cells reveal that autocrine WNT signaling may result in the deacetylation of $\beta$-catenin and activate the downstream signal transduction. In the absence of WNT ligands, acetylated $\beta$-catenin may mediate the phosphorylation and degradation of $\beta$-catenin under normal conditions. The deacetylation of $\beta$-catenin by HDAC6 modulates the in vitro proliferation of breast cancer cells through responding to WNT stimulation. The inhibition of WNT-elicited deacetylation of $\beta$-catenin exhibited a strong effect on the growth of breast cancer cells and it may provide a valid therapeutic approach in breast cancer.

\section{References}

1. Jemal A, Bray F, Center MM, Ferlay J, Ward E and Forman D: Global cancer statistics. CA Cancer J Clin 61: 69-90, 2011.

2. Chambers AF, Groom AC and MacDonald IC: Dissemination and growth of cancer cells in metastatic sites. Nat Rev Cancer 2: 563-572, 2002.

3. Clevers $\mathrm{H}$ : Wnt/beta-catenin signaling in development and disease. Cell 127: 469-480, 2006.

4. van Amerongen R, Mikels A and Nusse R: Alternative wnt signaling is initiated by distinct receptors. Sci Signal 1: re9, 2008.

5. MacDonald BT, Tamai K and He X: Wnt/beta-catenin signaling: components, mechanisms, and diseases. Dev Cell 17: 9-26, 2009.

6. Howe LR and Brown AM: Wnt signaling and breast cancer. Cancer Biol Ther 3: 36-41, 2004.

7. van de Wetering M, Barker N, Harkes IC, et al: Mutant E-cadherin breast cancer cells do not display constitutive Wnt signaling. Cancer Res 61: 278-284, 2001.

8. Bafico A, Liu G, Goldin L, Harris V and Aaronson SA: An autocrine mechanism for constitutive Wnt pathway activation in human cancer cells. Cancer Cell 6: 497-506, 2004.

9. Schlange T, Matsuda Y, Lienhard S, Huber A and Hynes NE: Autocrine WNT signaling contributes to breast cancer cell proliferation via the canonical WNT pathway and EGFR transactivation. Breast Cancer Res 9: R63, 2007.

10. Matsuda Y, Schlange T, Oakeley EJ, Boulay A and Hynes NE: WNT signaling enhances breast cancer cell motility and blockade of the WNT pathway by sFRP1 suppresses MDA-MB-231 xenograft growth. Breast Cancer Res 11: R32, 2009.

11. Nelson WJ and Nusse R: Convergence of Wnt, beta-catenin, and cadherin pathways. Science 303: 1483-1487, 2004.

12. Yang P, An H, Liu X, Wen M, Zheng Y, Rui Y and Cao X: The cytosolic nucleic acid sensor LRRFIP1 mediates the production of type I interferon via a beta-catenin-dependent pathway. Nat Immunol 11: 487-494, 2010.

13. Liu C, Li Y, Semenov M, et al: Control of beta-catenin phosphorylation/degradation by a dual-kinase mechanism. Cell 108: 837-847, 2002.

14. Ikeda S, Kishida S, Yamamoto H, Murai H, Koyama S and Kikuchi A: Axin, a negative regulator of the Wnt signaling pathway, forms a complex with GSK-3beta and beta-catenin and promotes GSK-3beta-dependent phosphorylation of beta-catenin. EMBO J 17: 1371-1384, 1998.

15. Kitagawa M, Hatakeyama S, Shirane M, et al: An F-box protein, FWD1, mediates ubiquitin-dependent proteolysis of beta-catenin. EMBO J 18: 2401-2410, 1999. 
16. Yost C, Torres M, Miller JR, Huang E, Kimelman D and Moon RT: The axis-inducing activity, stability, and subcellular distribution of beta-catenin is regulated in Xenopus embryos by glycogen synthase kinase 3. Genes Dev 10: 1443-1454, 1996.

17. Liu C, Kato Y, Zhang Z, Do VM, Yankner BA and He X: beta-Trcp couples beta-catenin phosphorylation-degradation and regulates Xenopus axis formation. Proc Natl Acad Sci USA 96: 6273-6278, 1999.

18. Wolf D, Rodova M, Miska EA, Calvet JP and Kouzarides T: Acetylation of beta-catenin by gapdh (CBP). J Biol Chem 277: 25562-25567, 2002.

19. Levy L, Wei Y, Labalette C, Wu Y, Renard CA, Buendia MA and Neuveut C: Acetylation of beta-catenin by p300 regulates beta-catenin-Tcf4 interaction. Mol Cell Biol 24: 3404-3414, 2004.

20. Zhu J, Coyne CB and Sarkar SN: PKC alpha regulates Sendai virus-mediated interferon induction through HDAC6 and $\beta$-catenin. EMBO J 30: 4838-4849, 2011.

21. Jho EH, Zhang T, Domon C, Joo CK, Freund JN and Costantini F: Wnt/beta-catenin/Tcf signaling induces the transcription of Axin2, a negative regulator of the signaling pathway. Mol Cell Biol 22: 1172-1183, 2002.

22. Roose J, Huls G, van Beest M, et al: Synergy between tumor suppressor APC and the beta-catenin-Tcf4 target Tcf1. Science 285: 1923-1926, 1999.
23. Li Y, Zhang X, Polakiewicz RD, Yao TP and Comb MJ: HDAC6 is required for epidermal growth factor-induced beta-catenin nuclear localization. J Biol Chem 283: 12686-12690, 2008.

24. Takahashi-Yanaga F and Sasaguri T: The Wnt/beta-catenin signaling pathway as a target in drug discovery. J Pharmacol Sci 104: 293-302, 2007.

25. Morin PJ, Sparks AB, Korinek V, Barker N, Clevers H, Vogelstein B and Kinzler KW: Activation of beta-catenin-Tcf signaling in colon cancer by mutations in beta-catenin or APC. Science 275: 1787-1790, 1997.

26. Fraga MF, Ballestar E, Villar-Garea A, et al: Loss of acetylation at Lys16 and trimethylation at Lys20 of histone $\mathrm{H} 4$ is a common hallmark of human cancer. Nat Genet 37: 391-400, 2005.

27. Lin YY, Kiihl S, Suhail Y, et al: Functional dissection of lysine deacetylases reveals that HDAC1 and p300 regulate AMPK. Nature 482: 251-255, 2012.

28. von Kries JP, Winbeck G, Asbrand C, et al: Hot spots in beta-catenin for interactions with LEF-1, conductin and APC. Nat Struct Biol 7: 800-807, 2000. 